\title{
Optimal Placement of DG Units for Loss Reduction in Distribution Systems Using One Rank Cuckoo Search Algorithm
}

\author{
MCV Suresh*1 and Belwin Edward J. ${ }^{2}$ \\ ${ }^{1}$ Department of Electrical and Electronics Engineering, \\ Sri Venkateswara College of Engineering, Tirupati, India \\ ${ }^{2}$ School of Electrical Engineering, Department of EEE, VIT University, \\ Vellore, India \\ mcvsuresh@gmail.com
}

\begin{abstract}
This paper presents a hybrid method to determine the optimal locations and sizes of $D G$ units in distribution networks using Fuzzy and one rank cuckoo search Algorithm (ORCS). The main objective functions are to reduce total power losses and to improve voltage profiles of power distribution networks. As major power losses are occurring on distribution networks, Keen interest is evinced to reduce them. Due to the increasing interest on renewable sources in recent times, the studies on integration of distributed generation to the power grid have rapidly increased. The Distributed Generation (DG) sources are added to the networks mainly to reduce the power losses by supplying a net amount of power. In order to minimize the line losses of power systems, it is equally important to define the size and location of local generation. In this paper fuzzy approach is used to find the optimal DG locations and one rank cuckoo search algorithm is used for optimal sizes of the DG units. The proposed method is tested on IEEE 15-bus, 33-bus and 69-bus test systems and the results are presented.
\end{abstract}

Keywords: Loss reduction, one rank cuckoo search algorithm, Fuzzy, radial distribution systems, DG unit

\section{Introduction}

Reduction of total power loss in distribution system is very essential to improve the overall efficiency of power delivery. This can be achieved by placing the optimal value of Distributed Generators at proper locations in radial distribution systems. DG units placed at optimal locations with optimum sizes to reduce the losses and to improve the voltages within the limits.

Application of Distributed Generators to the primary distribution feeders is a common practice in most of the countries. The objective of the Distributed Generators placement problem is to determine the locations and sizes of the Distributed Generators so that the power loss is minimized and annual savings are maximized.

Distributed generation has been a topic of research for the past few decades. A lot of study is carried out in this area. Dugan, R.C and Mc Dermott, T.E [1] defined the distributed generation as follows. Distributed generators that are interconnected to with utility distribution systems will generally be units smaller than $10 \mathrm{MW}$. Larger units are generally connected directly to transmission facilities and will most likely be commercial power producers. The units installed on distribution systems will typically be no larger than 1 or $2 \mathrm{MW}$. These would be installed mostly by the utility itself or by end users. This method of generation is commonly referred to as distributed generation (DG).

Received (October 26, 2017), Review Result (January 10, 2018), Accepted (January 11, 2018) 
In paper [2-3] the authors Celli and Pilo, discuss the necessity of knowing the power system impacts with DG and hence proposes a new software procedure, based on a genetic algorithm, capable of establishing the optimal distributed generation allocation on an existing MV distribution network, considering all the technical constraints, like feeder capacity limits, feeder voltage profile and three-phase short circuit current in the network nodes.

X. S. Yang and Suash Deb [4] developed an optimization technique. This algorithm is inspired by some species of a bird family called cuckoo because of their special life-style and aggressive reproduction strategy.

Multiple DG units were used by Naveen Jain [5] to minimize the power losses and evaluating the network capacity. The authors in [6] proposed a hybrid method i.e. Combined particle swarm optimization (PSOGA) to improve the voltage stability.Teaching Learning Based Optimization (TLBO) was proposed by Ravipudi venkata Rao [7] for solving continuous non-linear optimization problems. Optimal DG Placement and sizing by using index vector method and Flower pollination algorithm [8] to reduce the maximum power loss was proposed by dinakara Prasad reddy et al. Effect of load models in distribution system was presented in [9]. Comparison of different sensitivity based DG placement were proposed[10] by Murthy et al. An analytical approach based on exact loss formula has been presented in [11] to find the optimal size and location of DG however, voltage constraint has not been considered.

The authors in [12-15] uses new optimization algorithms like ant lion, whale optimization algorithms for DG allocation. The authors [16] in this paper uses particle swarm optimization algorithm is used for DG allocation. A new MLPSO was used in [17] for power loss reduction. A novel combined GA/PSO is presented in [18] for optimal DG placement on distribution systems.

In this paper, a novel method of optimal DG allocation using Fuzzy and new optimization algorithm called one rank Cuckoo search algorithm [19-20] is proposed for finding DG location and sizes. The results clearly indicate that DG reduces the electrical line loss.

\section{Problem Formulation}

The problem of DG allocation and sizing should be approached with caution. If DG units are connected at non-optimal locations, the system losses may increase. Studies have indicated that inappropriate locations or sizes of DG may lead to greater system losses than the ones in the existing network.

In this paper different methodologies to determine optimal locations and sizes of DG units to minimize the system real power loss are used. In achieving this objective some system constraints have to be satisfied. The distribution system considered is a balanced radial distribution system. The problem statement can be defined as

$$
\text { Objective Function=Min (TLP) }
$$

Where $T L P=\sum_{i=1}^{n} I_{i}^{2} R_{i}$ is the total real power loss of the radial distribution system. Subject to voltage constraint

$$
\left|v_{\text {imin }}\right| \leq\left|v_{i}\right| \leq\left|v_{\text {imax }}\right|
$$

Where $I_{i}$ is the current flowing through the $\mathrm{i}^{\text {th }}$ branch. $\mathrm{Ri}$ is the resistance of the $\mathrm{i}^{\text {th }}$ branch and $\mathrm{n}$ is the number of branches in the system. $\mathrm{V}_{\mathrm{imax}}$ and $\mathrm{V}_{\text {imin }}$ are the upper and lower limits on $i^{\text {th }}$ bus voltage.

\section{Optimal Dg Placement Using Fuzzy Approach}

This paper presents a fuzzy approach for determining optimal locations for DG units. 
Two objectives are considered while designing a fuzzy logic for identifying the optimal Distributed Generators locations. The two objectives are: (i) to minimize the real power loss and (ii) to maintain the voltage within the permissible limits. Voltages and Power loss indices of distribution system nodes are modeled by fuzzy membership functions. A set of rules are used in fuzzy inference system (FIS) for determining optimal DG unit locations. DG units are placed on the nodes with the highest suitability.

In distribution system with high losses and low voltage is highly ideal for placement of DG units. Whereas a low loss section with good voltage is not ideal for Distributed Generators placement. A set of fuzzy rules has been used to determine suitable Distributed Generators locations in a distribution system.

The real and reactive power losses are obtained from the base load flow. From this the loss sensitivity factor (LSF) values $\left(\frac{\partial \mathrm{P}_{\text {lineloss }}}{\partial \mathrm{Q}_{\text {eff }}}\right)$ can be calculated with the following equation.

$$
\frac{\partial \mathrm{P}_{\text {lineloss }}}{\partial \mathrm{Q}_{\text {eff }}}=\frac{\left(2 \times \mathrm{Q}_{\text {eff }}(\mathrm{j}) \times \mathrm{R}(\mathrm{k})\right)}{(\mathrm{V}(\mathrm{j}))^{2}}
$$

Where $Q_{\text {eff }}(j)$ is the total effective reactive power supplied beyond the bus ' $j$ '. These LSF values are linearly normalized into a $[0,1]$ range with the largest loss Sensitivity Factors having a value of 1 and the smallest one having a value of 0 . Loss sensitivity factor index (LSFI) value for $\mathrm{n}^{\text {th }}$ node can be obtained using below equation.

$$
\operatorname{LSFI}(n)=\frac{\operatorname{LSF}(n)-\operatorname{LSF}(\min )}{\operatorname{LSF}(\max )-\operatorname{LSF}(\min )}
$$

These LSFI values along with the p.u. nodal voltages are the inputs to the Fuzzy Inference System (FIS), which determines the node more suitable for DG unit placement.

In this work, two input and one output variables are selected. Input variable-1 is LSFI and Input variable-2 is the per unit nodal voltage $(\mathrm{V})$. Output variable is Distributed Generator suitability index (DSI).

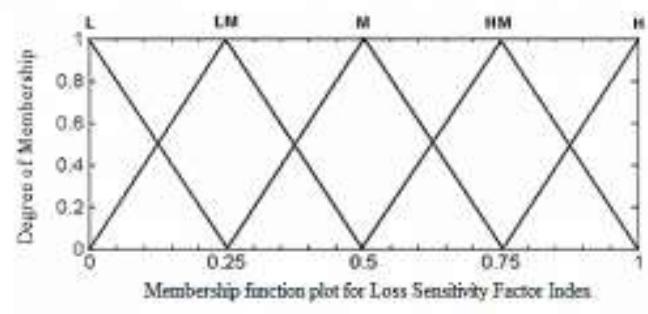

Figure 1. Membership Function Plot for LSFI

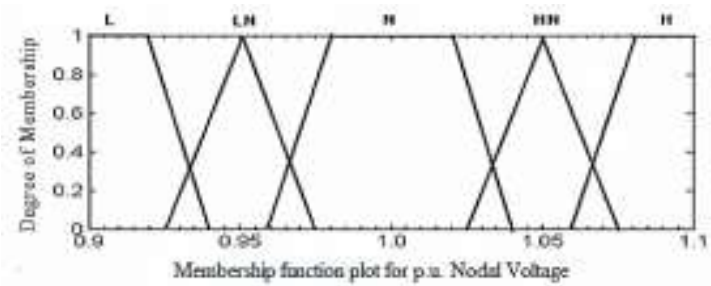

Figure 2. Membership Function Plot for p.u. Nodal Voltage 


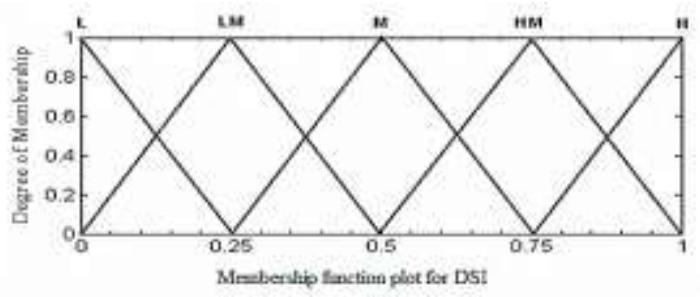

Figure 3. Membership Function plot for DSI

For the Distributed Generator allocation problem, rules are defined to determine the suitability of a node for Distributed Generator installation. Such rules are expressed in the following form:

IF premise (antecedent), THEN conclusion (consequent) for determining the suitability of Distributed Generator placement at a particular node, a set of multiple antecedent fuzzy rules has been established.

The inputs to the rules are the voltage and LSF indices and the output is the Distributed Generator suitability index. The rules are formed as shown in Table1. Here five membership functions are selected for LSFI. They are L, LM, M, HM and H. Five membership functions are selected for Voltage. They are $\mathbf{L}, \mathbf{L N}, \mathbf{N}, \mathbf{H N}$ and $\mathbf{H}$. Five membership functions are selected for DSI. They are L, LM, M, HM and H. These membership functions are shown in above Figures.

Table 1. Decision Matrix for Determining the Optimal DG Locations

\begin{tabular}{|c|l|r|r|r|r|c|}
\hline \multirow{2}{*}{\multicolumn{2}{|c|}{}} & \multicolumn{5}{|c|}{ Voltage Magnitudes } \\
\cline { 2 - 7 } AND & L & LN & N & HN & H \\
\hline \multirow{3}{*}{} & L & LM & LM & L & L & L \\
\cline { 2 - 7 } & LM & M & LM & LM & L & L \\
\cline { 2 - 7 } & M & HM & M & LM & L & L \\
\cline { 2 - 7 } & HM & HM & HM & M & LM & L \\
\cline { 2 - 7 } & H & H & HM & M & LM & LM \\
\hline
\end{tabular}

For finding best DG locations the output variable i.e. DSI values from FIS are arranged in descending order with corresponding bus numbers. Higher the DSI value, higher the probability of the best suitable location. The first ten DSI values with corresponding bus numbers are shown in Table 2.

Table 2. DSI Values in Descending Order

\begin{tabular}{|l|c|c|c|c|c|c|}
\hline \multirow{2}{*}{$\begin{array}{c}\text { s. } \\
\mathrm{N}\end{array}$} & \multicolumn{2}{|c|}{$\begin{array}{c}\text { IEEE } 15 \text { bus } \\
\text { system }\end{array}$} & \multicolumn{2}{c|}{$\begin{array}{c}\text { IEEE 33 bus } \\
\text { system }\end{array}$} & \multicolumn{2}{c|}{$\begin{array}{c}\text { IEEE 69 bus } \\
\text { system }\end{array}$} \\
\cline { 2 - 7 } & $\begin{array}{c}\text { DSI } \\
\text { Values }\end{array}$ & $\begin{array}{c}\text { Bus } \\
\text { no }\end{array}$ & $\begin{array}{c}\text { DSI } \\
\text { Values }\end{array}$ & $\begin{array}{c}\text { Bus } \\
\text { no }\end{array}$ & $\begin{array}{c}\text { DSI } \\
\text { Values }\end{array}$ & $\begin{array}{c}\text { Bus } \\
\text { no }\end{array}$ \\
\hline 1. & 0.5509 & 6 & 0.7646 & 6 & 0.7500 & 57 \\
\hline 2. & 0.5016 & 3 & 0.7622 & 28 & 0.7500 & 58 \\
\hline 3. & 0.5000 & 11 & 0.7500 & 29 & 0.5793 & 61 \\
\hline 4. & 0.2586 & 2 & 0.6209 & 30 & 0.4783 & 60 \\
\hline 5. & 0.2500 & 14 & 0.5223 & 9 & 0.4104 & 59 \\
\hline 6. & 0.2500 & 7 & 0.5077 & 13 & 0.3398 & 64 \\
\hline 7. & 0.2500 & 13 & 0.5029 & 10 & 0.2843 & 65 \\
\hline 8. & 0.2500 & 15 & 0.5000 & 3 & 0.2749 & 63 \\
\hline 9. & 0.2500 & 8 & 0.4678 & 8 & 0.2692 & 62 \\
\hline 10. & 0.2500 & 4 & 0.4267 & 5 & 0.2500 & 17 \\
\hline
\end{tabular}


Optimal DG locations are identified based on DSI values. For 15 bus system, nodes having DSI value greater than or equal to 0.5 are considered for optimal DG placement. Three candidate notes are selected for optimal DG placement. These nodes are 6, 3 and 11. For 33 bus system, nodes having DSI value greater than or equal to 0.6 are considered for optimal DG placement. Four candidate notes are selected for optimal DG placement. These nodes are 28, 6, 29 and 30. For 69 bus system, nodes having DSI value greater than or equal to 0.5 are considered for optimal DG placement. Three candidate notes are selected for optimal DG placement. These nodes are 57, 58 and 61.

\section{One Rank Cuckoo Search (ORCS) Algorithm}

Cuckoo Search algorithm [20] is inspired by some species of a bird family called cuckoo. Cuckoos lay their eggs in the nests and remove the existing eggs to increase the hatching probability of their eggs. On the other hand, some of the host birds are able to find the alien eggs and throw them out of their nests or build their new nests in new locations.

The following three idealized rules are considered

1. Each Cuckoo lays one egg at a time, and dumps it in a randomly chosen nest.

2. The best nests with high quality of eggs are carried over to the next generations.

3. The number of available host nests is constant, and the egg which is laid by a Cuckoo is discovered by the host bird with a probability of pa in the range of $[0,1]$. If a cuckoo's egg is very similar to a host's egg, then this cuckoo's egg is less likely to be discovered.

The one rank/combined evaluation and bound by best solution functionalities, have been added to the original cuckoo search algorithm, and used to draw the basic one rank cuckoo search algorithm. The proposed ORCS algorithm introduced one more parameter, one rank ratio update trigger $t_{o r}$, in addition to the two parameters employed by the original CS algorithm, population size and abandon rate $\mathrm{p}_{\mathrm{a}}$.

In the proposed algorithm one rank/combined evaluation is used to generate new solutions using Levy flights, replaces a fraction of them, and finally evaluates and ranks their fitness at once. A one rank ratio $r_{o r}$ is initiated by 1 , to allow the proposed algorithm to combine all the explorations and exploitations, until it fails to find better nests for $t_{\text {or }}$ iterations, to trigger a gradual decrease of the one rank ratio as shown below, where $t$ is the iteration number and $\mathrm{D}$ is the number of objective function dimension.

$$
\mathrm{r}_{\mathrm{or}}^{\mathrm{t}+1}=\mathrm{r}_{\mathrm{or}}^{\mathrm{t}} \times 1-0.5 / \mathrm{D}
$$

The proposed algorithm enforces the integrity over an out of constraints solution by replacing its invalid dimensions by the corresponding dimensions drawn from randomly selected solutions among the current best solutions by using Bound by Best Solutions. A ratio of the replaced dimensions is utilizing the current best solutions found so far, and the rest is being randomly drawn by further exploring the search space. A bound by best ratio is defined as below equation.

$$
\mathrm{r}_{\mathrm{bbb}}=1-1 / \sqrt{\mathrm{D}}
$$

Algorithm for DG Placement and Sizing Using Fuzzy Approach and one rank CS Algorithm

1. Initialize all the parameters and control parameters of CS algorithm. They are $\mathrm{DG}_{\min }=60 \mathrm{kVA}, \mathrm{DG}_{\max }=3000 \mathrm{kVA}$, population nop=15, number of $\mathrm{dg}$ units ndg, discovery rate of alien egss/solutions probability $\mathrm{p}_{\mathrm{a}}=0.25, \mathrm{t}_{\mathrm{or}}=0.1$.

2. Initially [pop x ndg] population is generated randomly within the limits. Nest $(\mathrm{i},:)=\mathrm{DG}_{\min }+\left(\mathrm{DG}_{\max }-\mathrm{DG}_{\min }\right)$. * $\operatorname{rand}\left(\operatorname{size}\left(\mathrm{DG}_{\min }\right)\right)$ 
3. By placing all the nests at the respective DG location and find the fitness i.e. total real power loss. The same procedure is repeated for the pop number of nests. Get the current best of fitness function.

4. Start iterations

5. Generate new set of nests or solutions randomly but keep the current best

Nest $(i,:)=$ Nest $(i,:)+$ step size.*randn $(\operatorname{size}(s))$

6. A fraction of worse nests are replaced with the probability pa and new nest is discovered by

$$
\begin{gathered}
\mathrm{K}=\text { rand }(\text { size }(\text { Nest }))>\text { pa } \\
\text { Step size= }=\text { rand*(Nest }(\operatorname{rand}(\mathrm{n}),:)-\text { Nest }(\operatorname{rand}(\mathrm{n}),)) \\
\text { New_nest=Nest + stepsize. }{ }^{* K}
\end{gathered}
$$

7. Evaluate new population obtained from bound by best solution procedure and find out fitness value.

8. Find the best nest corresponding to best fitness value so far.

9. Increment Iterations.

10. The DG unit sizes corresponding to minimum fitness (Best Nest) gives the optimal DG unit sizes. Repeat till end of iterations and get the best solution.

\section{Results}

The proposed algorithm is applied to IEEE 15, 33 and 69 bus systems. Optimal DG locations are identified based on the DSI values. The optimal locations for 15, 33 and 69bus system are three, four and three locations respectively. Distributed Generator sizes in optimal locations, total real power losses before and after compensation are shown in Tables 3, 4 and 5 .

Table 3. Results of 15-Bus System

\begin{tabular}{|c|c|}
\hline Bus No. & DG unit size in kVA \\
\hline 6 & 546 \\
\hline 3 & 769 \\
\hline 11 & 364 \\
\hline $\begin{array}{c}\text { Minimum Bus Voltage } \\
\text { in p.u. (before) }\end{array}$ & 0.9445 \\
\hline $\begin{array}{c}\text { Minimum Bus Voltage } \\
\text { in p.u. (after) }\end{array}$ & 0.9923 \\
\hline $\begin{array}{c}\text { Total power loss in kW } \\
\text { (before) }\end{array}$ & 61.7933 \\
\hline $\begin{array}{c}\text { Total power loss in kW } \\
\text { (after) }\end{array}$ & 4.6687 \\
\hline \% of loss reduction & 92.44 \\
\hline
\end{tabular}


Table 4. Results of 33-Bus System

\begin{tabular}{|c|c|}
\hline Bus No. & $\begin{array}{c}\text { DG unit size in } \\
\text { kVA }\end{array}$ \\
\hline 6 & 1764 \\
\hline 28 & 91 \\
\hline 29 & 139 \\
\hline 30 & 0.8785 \\
\hline $\begin{array}{c}\text { Minimum Bus Voltage } \\
\text { in p.u. (before) }\end{array}$ \\
\hline $\begin{array}{c}\text { Minimum Bus Voltage } \\
\text { in p.u. (after) }\end{array}$ \\
\hline $\begin{array}{c}\text { Total power loss in kW } \\
\text { (before) }\end{array}$ & 369.2543 \\
\hline $\begin{array}{c}\text { Total power loss in kW } \\
\text { (after) }\end{array}$ & 64.0354 \\
\hline \% of loss reduction & 82.66 \\
\hline
\end{tabular}

Table 5. Results of 69-Bus System

\begin{tabular}{|c|c|}
\hline Bus No. & DG unit size in kVA \\
\hline 57 & 342 \\
\hline 58 & 92 \\
\hline 61 & 1927 \\
\hline $\begin{array}{c}\text { Minimum Bus Voltage } \\
\text { in p.u. (before) }\end{array}$ & 0.9092 \\
\hline $\begin{array}{c}\text { Minimum Bus Voltage } \\
\text { in p.u. (after) }\end{array}$ & 0.9727 \\
\hline $\begin{array}{c}\text { Total power loss in kW } \\
\text { (before) }\end{array}$ & 225.0225 \\
\hline $\begin{array}{c}\text { Total power loss in } \mathrm{kW} \\
\text { (after) }\end{array}$ & 88.12 \\
\hline$\%$ of loss reduction & 264 \\
\hline
\end{tabular}

The results show that $92.44 \%$ reduction in power loss for 15-bus system, $82.66 \%$ reduction in power loss for 33 -bus system and $88.12 \%$ reduction in power loss for 69 -bus system is possible as shown in tables and bus voltages are also improved substantially.

\section{Conclusions}

In this paper a new two stage methodology is proposed for DG placement problem. In stage I candidate locations for DG unit placement are determined and in stage II sizes of DG units at these locations are determined for minimum power loss. To determine the locations Fuzzy approach is developed. The proposed fuzzy approach is capable of determining the optimal DG locations based on the fuzzy rule base. The proposed one rank CS Algorithm method iteratively searches the optimal DG sizes at these locations effectively for the maximum power loss reduction. The validity of the proposed method is proved from the comparison of the results of the proposed method with and without DG. By installing DGs at all the potential locations, the total power loss of the system has been reduced drastically and the voltage profile of the system is also improved. 


\section{References}

[1] R.C. Dugan and T.E. McDermott, "Distributed Generation”, Industry Applications Magazine, IEEE, vol. 8 , issue 2, (2002), pp. $19-25$.

[2] G. Celli and F. Pilo, "Optimal distributed generation allocation in MV distribution networks", Proceedings of the $2001 \mathrm{IEEE/PES} \mathrm{conference} \mathrm{on} \mathrm{power} \mathrm{industry} \mathrm{computer} \mathrm{application,} \mathrm{(2001),} \mathrm{pp.} 81$ 86.

[3] G. Celli and F. Pilo, "MV network planning under uncertainties on distributed generation penetration", Proceedings of the IEEE 2001 Power Engineering Society Summer Meeting, vol. 1, (2001), pp. $485-490$

[4] X.S. Yang and S. Deb, "Cuckoo search via Levy flights", World Congress on Nature \& Biologically Inspired Computing (NaBIC 2009), December 2009, India, IEEE Publications, USA, (2009), pp. 210214.

[5] N. Jain, S.N. Singh and S.C. Srivastava, "Particle Swarm Optimization Based Method for Optimal Sitting and Sizing of Multiple DGs", 16th national power system conference, (2010).

[6] M. Hasan Moradi and M. Abedini Ghosh, "A Combination of Genetic Algorithm and Particle Swarm Optimization for Optimal DG Location and Sizing in Distribution Systems", International Journal of Electrical Power and Energy systems, vol. 34, issue 8, (2012), pp. 66-74.

[7] R. Venkata Rao, V. J. Savsani and D.P. Vakharia. "Teaching Learning Based Optimization: An Optimization Method for Continuous Non-Linear Large Scale Problems", International Journal of Electrical Power and Energy systems, vol. 183, issue 1, (2012), pp. 1-15.

[8] P. Dinakara Prasad Reddy, V.C. Veera Reddy and T. Gowri Manohar, "Application of flower pollination algorithm for optimal placement and sizing of distributed generation in Distribution systems", Journal of Electrical Systems and Information Technology, ISSN 2314-7172, vol. 3, Issue 1, (2016), pp. 14-22.

[9] Q. Kejun, Z. Chengke, A. Malcolm and Y. Yue, "Effect of load models on assessment of energy losses in distribution generation planning", Electr Power Res, vol. 12, (2011), pp. 43-50.

[10] V.V.S.N. Murthy and A. Kumar, "Comparison of optimal DG allocation methods in radial distribution systems based on sensitivity approaches", International Journal of Electrical Power \& Energy Systems, ISSN 0142-0615, vol. 53, (2013), pp. 450-467.

[11] N. Acharya, P. Mahat and N. Mithulananthan, "An analytical approach for DG allocation in primary distribution network", International Journal of Electrical Power \& Energy Systems, vol. 28, (2006), pp. 669-678.

[12] D.P. P. Reddy, V.C. Veera Reddy and T. Gowri Manohar. "Ant Lion optimization algorithm for optimal sizing of renewable energy resources for loss reduction in distribution systems", Journal of Electrical Systems and Information Technology, (2017).

[13] D.P. P. Reddy, V.C. Veera Reddy and T. Gowri Manohar, "Renewable energy resources placement in distribution systems by whale optimization algorithm", International Journal of Computer Science and Information Security, vol. 14, no. 12, (2016), p. 489.

[14] P. D. P. Reddy, V.C. Veera Reddy and T. Gowri Manohar, "Whale optimization algorithm for optimal sizing of renewable resources for loss reduction in distribution systems", Renewables: Wind, Water, and Solar, vol. 4, no. 1, (2017), p. 3.

[15] V.C. Veera Reddy and T. Gowri Manohar, "Optimal renewable resources placement in distribution networks by combined power loss index and Whale optimization algorithms", Journal of Electrical Systems and Information Technology, (2017).

[16] S. Kansal, V. Kumar and B.Tyagi, "Optimal placement of different type of DG sources in distribution networks", International Journal of Electrical Power \& Energy Systems, vol. 53, (2013), pp. 752-760.

[17] P. Kayal and C.K. Chanda, "Placement of wind and solar based DGs in distribution system for power loss minimization and voltage stability improvement", International Journal of Electrical Power \& Energy Systems, vol. 53, (2013), pp. 795-809.

[18] M.H. Moradi and M., Abedini, "A combination of genetic algorithm and particle swarm optimization for optimal DG location and sizing in distribution systems", International Journal of Electrical Power \& Energy Systems, vol. 34, (2012), pp. 66-74.

[19] P. Reddy and D. Prasad, "Sensitivity based capacitor placement using cuckoo search algorithm for maximum annual savings", IOSR Journal of Engineering, vol. 4, no. 4, (2014), p. 6.

[20] T. T. Nguyen, D.N. Vo and W. Ongsakul, "One rank cuckoo search algorithm for short-term hydrothermal scheduling with reservoir constraint", PowerTech, 2015 IEEE Eindhoven. IEEE, (2015). 